Relations industrielles

Industrial Relations

\title{
La rupture du contrat de travail
}

\section{Georges-Michel Giroux}

Volume 4, numéro 9, mai 1949

URI : https://id.erudit.org/iderudit/1023964ar

DOI : https://doi.org/10.7202/1023964ar

Aller au sommaire du numéro

Éditeur(s)

Département des relations industrielles de l'Université Laval

ISSN

0034-379X (imprimé)

1703-8138 (numérique)

Découvrir la revue

Citer cet article

Giroux, G.-M. (1949). La rupture du contrat de travail. Relations industrielles / Industrial Relations, 4(9), 83-87. https://doi.org/10.7202/1023964ar

Tous droits réservés (C Département des relations industrielles de l’Université Laval, 1949
Ce document est protégé par la loi sur le droit d'auteur. L'utilisation des services d'Érudit (y compris la reproduction) est assujettie à sa politique d'utilisation que vous pouvez consulter en ligne.

https://apropos.erudit.org/fr/usagers/politique-dutilisation/ 
aussi nombreuses que possible, il est par conséquent fort désirable que les ouvriers puissent eux aussi, du fruit de leur épargne, participer à la constitution du capital national.

Nombre d'hommes, industriels comme vous, catholiques, et non catholiques aussi, ont, en maintes circonstances, expressément déclaré que la doctrine sociale de l'Eglise - et elle seule - est en mesure de fournir les éléments essentiels pour une solution de la question sociale. Assurément la mise en oeuvre et l'application de cette doctrine ne peuvent être l'ouvrage d'un jour. Sa réalisation exige de tous les participants une sagesse clairvoyante et prévoyante, une forte dose de bon sens et de bon vouloir. Elle réclame d'eux surtout une réaction radicale contre la tentation de chercher chacun son propre avantage aux dépens des autres participants - quelle que soit la nature et la forme de leur participation - et au détriment du bien commun. Elle requiert enfin un désintéressement tel que, seule, une authentique vertu chrétienne, soutenue par l'aide et la grâce de Dieu, peut l'inspirer.

C'est pour attirer cette aide et cette grâce sur votre Union, sur son développement interne et sur son rayonnement au dehors, particulièrement dans les pays qui, tout catholiques qu'ils sont, ont pourtant besoin de s'ouvrir plus largement à la pensée sociale de l'Eglise, que Nous vous donnons, dans toute l'effusion de Notre coeur, à vous tous et à votre Association, sous le puissant patronage de la Mère du divin amour, Notre Bénédiction Apostolique.

\section{LA RUPTURE DU CONTRAT DE TRAVAIL}

\section{Georges-Michel Giroux}

L'abrogation de la Loi des maitres et des serviteurs (S.R.Q., 1941, c. 328), décrétée par le statut 13 Geo. VI, c. 69 , a modifié les règles sur la résiliation du contrat de travail. De multiples causes peuvent éteindre les obligations résultant de ce contrat, citons: l'accord de volontés, le dècès du salarié (C.c. a. 1668). La volonté unilatérale d'une des parties peut aussi y mettre fin, il y a alors rupture du contrat de travail.

Pour traiter de la rupture du contrat, il faut analyser les règles sur sa durée et celles sur le préavis de rupture, il faut aussi examiner le caractère des dispositions sur le délai-congé et l'effet de la réglementation du travail sur ce sujet.

\section{I - La durée du contrat}

Toute personne peut, quand il lui plaît, mettre fin à un contrat de travail qui la lie à une autre, c'est l'évidence même; un patron ne peut être forcé à embaucher un salarié et ce dernier, à travailler pour un employeur; nemo ad actum cogi potest. Le Conseil canadien des relations ouvrières, sous le régime de la Loi sur les relations industrielles et sur les enquêtes visant les différends du travail (Canada, 11-12 Geo. VI, c. 54), peut bien ordonner à un employeur de reprendre à son emploi des salariés congédiés indûment (a. 40,2); mais le patron, s'il ne se conforme pas à l'ordonnance, n'en- court que des pénalités (a. 40, 3). Un salarié, même sur l'ordre du Conseil canadien des relations ouvrières, ne peut travailler pour un employeur contre le gré de ce dernier.

Cependant le législateur a déterminé le temps où le contrat de travail pouvait être légalement rompu par la volonté d'une seule partie et a assuré à la partie lésée une indemnité quand l'autre partie en a provoqué abusivement la rupture. La loi, nous sommes ici en droit privé et non en droit public, veut assurer le respect de ce qui a été convenu; elle garantit la subsistance de l'engagement pour le terme, déterminé ou déterminable, fixé par les parties. L'étude sur la rupture du contrat de travail entraîne l'examen des règles légales sur la durée.

Les parties peuvent arrêter la durée de ce contrat:

a) pour une période déterminée (C.c. a. 1667 );

b) pour la durée d'une entreprise déterminée (Id.);

c) pour un temps indéterminé (Id. a. 1668);

d) à l'essai.

Les salariés dans la construction d'immeubles, s'engagent ordinairement pour la durée d'une entreprise déterminée; les briqueteurs louent leurs services pour le temps nécessaire à l'érection des murs. 
Notre Code Civil, tout comme le Code français, à l'origine, ne prévoyait pas l'engagement pour un laps de temps indéterminé, mais seulement celui pour une période fixe. L'économie et les moeurs d'alors permettaient d'assurer aux salariés la sécurité de leur emploi. Encore au début $\mathrm{du}$ siècle, dans les entreprises commerciales, immédiatement après l'inventaire, on parlait d'engagements et l'on signait pour l'année. Mais ces procédés n'existent presque plus et l'employeur actuellement ne garantit pas de durée d'emploi.

L'on ne peut ètre engagé pour un terme déterminé, qu'en autant que cela a été convenu. Il ne faut pas confondre les stipulations sur la durée, avec celles sur la périodicité de la paie et celles sur le mode de fixation du salaire. L'engagement, à tant par année ${ }^{1}$ n'est pas nécessairement un engagement pour un an, mais en est un où le salarié aura le droit de réclamer pour son travail une rémunération proportionnelle à celle fixée pour l'année.

La périodicité de la paie ne comporte pas in se de détermination de la durée de l'emploi ${ }^{2}$. C'est ce qu'exprime le législateur au second paragraphe de l'article 1668 du Code $^{3}$, en se référant « au domestique, serviteur, compagnon ou journalier engagé à la semaine, au mois ou à l'année, mais pour un laps de temps indéfini...»

Le contrat conclu pour une durée fixe se renouvelle automatiquement à son échéance pour un semblable terme; c'est la tacite reconduction édictée par l'article 1667 du Code Civil.

Le Code, avant la modification de l'article 1668, ignorait le contrat pour un laps de temps indéterminé. «On a interprété, déclare $\mathrm{M}$. le juge Rinfret ${ }^{4}$, la règle qui veut que le louage de services personnels ne puisse être que pour un temps limité comme voulant dire qu'un contrat de ce genre ne peut être fait pour toute la vie du locateur, ou pour une période de temps qui équivaudrait à une location permanente. Mais la doctrine et la jurisprudence n'ont jamais compris qu'un louage de services personnels ne pouvait être fait pour un temps indéterminé. La seule conséquence d'un contrat de ce genre est que l'une des parties peut s'en libérer en donnant un avis de congé raisonnable.»

Actuellement, l'engagement pour une période indéterminée est le fait normal; dans le commerce,

(1) Cour Suprême, Asbestos Corporation Ltd. c. Cook, 1933, C.L.R., 86.

(2) Cour Suprême, Hanover Fire Insurance c. Stewart 1936, C.L.R., 137.

(3) Ce paragraphe a été ajouté par 13 Geo. VI (1949), c. 69.

(4) Cause Asbestos, 91. le salaire est établi à la semaine et, dans l'industrie, à l'heure; la périodicité de la paie est la semaine ou la quinzaine.

$$
\text { II - Le délai-congé }
$$

Une partie peut rompre en tout temps un contrat de travail, mais pour le résilier légalement il faut en principe que préavis en soit donné à son contractant par celui qui provoque la rupture de contrat. Quand ce préavis doit-il être donné et quel est le délai déterminé par la loi pour l'avis de rupture ? La réponse varie avec la durée des contrats:

a) Contrat à l'essai: Cet engagement, vu sa nature, peut être rompu en tout temps et sans préavis, par l'une ou l'autre des parties.

b) Contrat pour une entreprise déterminée: Ce louage de services prend fin avec l'achèvement des travaux; l'engagement d'un joueur de hockey se termine avec la fin de la saison.

c) Contrat pour une période déterminée: Le contrat expire avec l'arrivée du terme sans qu'il $\mathrm{y}$ ait lieu à avis de rupture. Si le ler janvier 1949 je me suis engagé pour un an, mes services cessent automatiquement et sans avis le ler janvier 1950. Mais si l'emploi continue, le contrat se trouve automatiquement renouvelé par tacite reconduction, et le délai-congé est soumis à des règles spéciales qui seront ci-après étudiées.

d) Contrat pour une période indéterminée: Ce contrat n'était pas prévu au Code, mais a été reconnu par les tribunaux. M. le juge Rinfret a décidé dans la cause Asbestos (voir ci-haut), que ce contrat pouvait être rompu par un avis donné dans un délai raisonnable.

Evidemment il s'agit ici de salariés travaillant régulièrement, de salariés permanents, et non des auxiliaires et aides appelés en surcroìt pour une période déterminée ou déterminable.

On appliquera, comme nous y invite la Cour Suprême dans l'affaire Hanover (1936, C.L.R., 137), les règles établies par le Code pour le délaicongé prévu pour la location d'immeubles (C.c. a. 1642 et 1657 ). Citons ces textes:

« 1657. Lorsque le terme du bail est incertain, verbal, ou présumé, tel que réglé en l'article 1608 , aucune des parties n'y peut mettre fin sans en signifier congé à l'autre avec un délai de trois mois, si le loyer est payable par termes de trois mois ou plus; si le loyer est payable à des termes plus rapprochés que trois mois, le délai du congé est réglé suivant l'article 1642 .

Le tout néanmoins sujet aux dispositions de ce dernier article et des articles 1608 et 1653. »

«1642. Le bail d'une maison ou de partie d'une maison, lorsque la durée n'en est pas fixée, est censé fait à 
l'année, finissant au premier jour de mai de chaque année, lorsque le loyer est de tant par an;

Pour un mois, lorsque le loyer est de tant par mois;

Pour un jour, lorsque le loyer est de tant par jour.

Si rien ne constate un montant de loyer pour un terme fixe, la durée du bail est réglée par l'usage du lieu. »

Le délai-congé pour le bail de maisons se trouve conditionné par la périodicité du loyer; en transposant ces règles dans le domaine du contrat de travail, le délai-congé, pour rompre ce dernier contrat lorsqu'il est à laps de temps indéterminé, sera donc la période de la paie.

Conséquemment la partie provoquant la résiliation d'un tel contrat doit donner à l'autre un préavis équivalent à une période de paie, soit donc un mois, une quinzaine ou une semaine.

Il faut se souvenir qu'il n'y a pas toujours équivalence entre la périodicité de la paie et le mode de sa fixation.

Des salariés peuvent recevoir leur paie au mois, à la semaine, bien que leur rémunération soit établie à l'heure, aux pièces ou à commission. Ce que le législateur prend en considération pour déterminer le délai-congé, c'est la périodicité de la paie et non le mode de fixation du salaire. Ainsi le salarié payé à l'heure sans garantie d'emploi, peut avoir droit à un délai-congé d'une semaine.

Mais en suivant cette règle, il faut se souvenir que certains salariés ne reçoivent pas à chaque paie tout ce qui leur est dû. Ainsi, en plus de la prestation périodique, ils peuvent avoir droit à des commissions, à des bonis payables annuellement. Il faudra alors considérer que leur salaire est annuel comme nous y a invité la Cour suprême dans l'affaire Hanover, et qu'ils ont droit à un délai-congé de trois mois (C. c. a. 1657).

Un régime spécial a été établi pour certains salariés par la modification apportée à l'article 1668 C. c., nous l'étudierons ci-après.

\section{e) Contrat pour une durée déterminée auto- matiquement prorogée.}

Le contrat conclu pour un terme déterminé se renouvelle automatiquement à son échéance pour une période de même durée, c'est la tacite reconduction prévue à l'article 1667 C.c. Citons M. le juge Rinfret dans la cause Hanover: ${ }^{5}$

«Et si le contrat était, comme nous le décidions (dans l'affaire Asbestos), pour une période indéterminée, il ne pourrait être question de tacite reconduction. En effet, comme le fait remarquer Migneault, Droit civil canadien, vol. 7, p. 371: «Pour qu'il y ait lieu à tacite reconduction, il faut qu'il y ait un terme convenu ou présumé pour la durée du service. »

(5) Id.
«La tacite reconduction n'a lieu que si les relations des parties persistent après l'expiration de la date fixée au bail de services; dans le cas d'un louage pour une période indéterminée, le cas ne saurait se présenter. »

Quand expire le contrat de travail prorogé par tacite reconduction? Il n'expire pas de plein droit, il peut prendre fin à la date du renouvellement mais à la condition qu'un préavis de rupture ait été donné. Citons encore M. le juge Rinfret dans la cause Hanover:

«But it also means that, once having been prolonged beyond the term originally fixed in the contract ( «terme conventionnel $\gg$ ), it was no longer a contract which, by its very terms, was to terminate at a fixed date mutually agreed upon; it became a contract which, by law, was presumed to be prolonged for another period of time fixed by the law itself, and with the proviso that it would terminate upon one or the other party giving a notice «within the delay required by the law ».

Le contrat de travail sujet à reconduction tacite ne s'éteint pas de plein droit, il ne peut prendre fin qu'à la date de son renouvellement, mais à la condition expresse qu'un préavis ait été donné par la partie qui en provoque la rupture.

Quel est le délai de ce préavis ? Nous revenons ici aux règles des articles 1642 et 1657, le délai est encore la période de la paie.

Si le délai-congé est le même pour le contrat à terme fixe prorogé que pour le contrat à durée indéterminée, la rupture dans le premier cas ne peut avoir lieu quà la date du renouvellement. Dans le second cas, elle a lieu en tout temps.

\section{f) Contrat des salariés visés à l'article 1668 .}

Le législateur a ajouté, à la dernière session, le paragraphe suivant à l'article 1668 C.c.:

«Dans le cas d'un domestique, serviteur, compagnon ou journalier engagé à la semaine, au mois ou à l'année, mais pour un laps de temps indéfini, il peut être mis fin au contrat par avis, de l'une des parties à l'autre, d'une semaine, si l'engagement est à la semaine; de deux semajnes, si l'engagement est au mois; d'un mois, si l'engagement est à l'année. »

Cet article ne s'applique pas au personnel de maîtrise, aux techniciens, aux salariés de bureau, aux commis voyageurs ni aux commis de magasin, mais il régit les bonnes et les personnes au service personnel d'un patron et aussi les personnes faisant un travail manuel spécialisé ou non, travaillant dans des établissements industriels, commerciaux ou autres. 
L'article 1668 s'applique aux salariés y visés qu'en autant qu'ils sont «engagés à la semaine, au mois ou à l'année, mais pour un laps de temps indéfini.» Le législateur ne considère pas néccssairement le mode de fixation du salaire, v.g. ceux payés à l'heure, au jour, aux pièces, à forfait ou à commission, et conséquemment cet article régit les salariés y visés sans considération du mode d'établissement de leur paie, soit à l'heure, aux pièces ou autrement.

La loi vise ceux qui travaillent de semaine $\mathrm{en}$ semaine, de mois en mois, ou encore d'année en année, soit donc ceux qui se sont engagés pour un temps fixe, v.g. semaine, mois ou an, et dont le contrat s'est trouvé renouvelé par tacite reconduction (C.c. a. 1667) et ceux dont le terme du contrat, à défaut de convention expresse, est présumé, suivant l'article 1642 C.c., la période de paie.

Ce nouveau texte régit donc les salariés qui travaillent régulièrement, les salariés ayant un emploi constant et non les auxiliaires appelés à aider pour un surcroit de travail.

L’employeur des salariés visés à l'article 1668 doit, avant de les remercier de leurs services, leur donner un préavis d'une semaine, de deux semaines ou d'un mois.

En conflit avec l'article 1667, l'article 1668 aura priorité. S'il appert que, quoique ayant conclu un contrat pour un an, les parties entendaient le laisser se renouveler automatiquement, l'une d'elles pourra y mettre fin en tout temps sur préavis d'un mois. Les salariés visés par l'article 1668 ne peuvent bénéficier des avantages de la prorogation prévue à l'article 1667.

\section{g) Autre cas.}

Le délai-congé est donc en relations avec la durée du contrat de travail. Souvent le terme en est exprès; mais, quand il ne l'est pas, il faut déterminer, suivant les circonstances, ce que les parties ont entendu établir comme durée.

Les règles précédentes régissent principalement ceux qui ont un emploi stable, ceux qui sont censés travailler à chaque jour de travail. Mais, dans l'industrie, beaucoup de salariés sont appelés comme auxiliaires à aider pour un surcroît de travail ou encore pour accomplir une tâche déterminée, v.g. décharger un wagon. Il y a donc des cas où réellement il y a engagement pour un travail arrêté ou encore où il y a engagement à l'heurc, dans ce dernier cas le délai-congé étant d'une heure.

\section{III - Les consequences}

Pas de renvoi ou d'abandon de travail sans préavis, tel que prévu par la loi, de la partie qui provoque la rupture du contrat, c'est la règle générale. Si le délai-congé n'est pas donné ou s'il est insuffisant, la partie lésée a droit de s'en plaindre et de réclamer de l'autre partie les dommages qu'elle subit. Ces dommages pour le salarié représentent ordinairement le salaire qu'il aurait gagné durant le délai-congé.

Chez nous on n'admettrait pas le renvoi abusif reconnu en droit français. Explicitant la théorie de l'abus de droit, le législateur français considère que l'employeur, même en respectant le délaicongé, peut être en faute de licencier un salarié; il l'oblige, en tel cas, à payer au salarié une indemnité qui tient compte de son âge, de sa compétence, de son ancienneté, de la possibilité d'occuper un poste identique dans la hiérarchie du travail, (C. f., t. 1, a. 23). ${ }^{7}$

Mais le contrat de travail, comme toute autre convention, peut être rompu pour cause, alors il n'y a plus d'obligation de préavis et conséquemment il n'existe aucun recours de la partie lésée par la rupture. Citons Me P. Beaulac ${ }^{s}$ :

«Le renvoi d'un employé pourra avoir été justifié par ses manquements graves. Tels seront le cas de l'employé qui s’est enivré pendant les heures de services", celui du rédacteur de journal qui a publié des articles contraires à la politique du journal ${ }^{10}$, celui du commis de magasin qui s'est absenté sans permission ${ }^{11}$, celui du gerant qui s'est rendu coupable d'insolence ou d'insurbordination envers les directeurs de la compagnie ${ }^{12}$, ou qui s’est approprié des deniers appartenant à son patron ${ }^{13}$, celui de l'employé qui refuse de se faire vacciner comme l'exige la loi ${ }^{14}$, celui du commis-voyageur qui, sans raison valable, se retuse à certains voyages ${ }^{15}$. Il ne saurait être question ici d'un avis de congé ».

(6) P. Bcaulac, La rupture du contrat de travail, 2 R. du B., 306.

(7) Précis de législation industrielle, 1947, Rouast \& Durand, no 346 \& s.

(8) Rupture du contrat de travail, 2 R. de B. 309.

(9) Provost c. Standard Foundry \& Machinery Co. (1915) 21 R.L., 433.

(10) Bélanger c. Bélanger, (1895) 18 L.N., 354 ou 24 S.C.R., 678.

(11) Charbonneau c. Benjamin, (1858) 2 J., 103.

(12) Dick c. Canada Jute Co. (1890) 34 J. 73 ou (1890) 18 R.L., 555.

(13) Prévost c. Standard Foundry \& Machinery Co. précité. Webster c. G.T.R. 1 J. 223

(14) Archambault c. Gazette Printing Co. (1836) 9 L.N.. 11.

(15) Prévost c. Gauthier (1878) 1 L.N., 289. 


\section{IV - La règlementation}

Quel est le caractère de notre législation sur le délai-congé ? Nous la trouvons, ne l'oublions pas, dans le Code civil, au titre des contrats. Et la règlementation des conventions établie par le Code n'est que du droit supplétif; elle ne s'applique que lorsque les parties n'ont pas établi conventionnellement un autre régime, puisqu'il n'y a pas ici matière concernant l'ordre public ou les bonnes moeurs (C.c. a. 13).

Conséquemment, les parties peuvent établir à volonté les règles sur le délai-congé, et cette règlementation conventionnelle fait obstacle aux règles supplétives du Code. Me $\mathrm{P}$. Beaulac ${ }^{18}$, dans son étude, signale bien cette règle: «A défaut de raison suffisante, il faudra un avis de congé qui soit conforme à la convention expresse ou implicite entre les parties. Les règlements de la municipalité, ceux de l'industrie ou de l'usine, lusage ou la coutume pourront, suivant le cas, suppléer à la convention. »

Les dispositions établies par la Loi des mâ̂tres et serviteurs (S.R.Q., 1941, c. 328), comportaient une règlementation pénale, et donc des règles qu'il n'était pas au pouvoir des parties de modifier. Si l'employé quittait son emploi sans donner l'avis prévu à ce statut, il pouvait être cité en correctionnelle, mis à l'amende et envoyé en prison; les contractants ne pouvaient pas, parce que d'ordre public, se dispenser de l'avis de congé prévu par cette loi. Mais le statut 13 Geo. VI, c. 69 a abrogé cette loi ${ }^{1 \pi}$ et en a introduit les dispositions au Code civil.

(16) Id., 308.

(17) La Loi des maìtres et serviteurs ne s'appliquait pas aux cités et villes qui avaient passé des règlements régissant les relations entre les maîtres et les serviteurs (Id. a. 2). Le statut 13 Geo. VI, c. 69 a abrogé non seulement cette loi, mais encore les règlements adoptés sous son régime. Me B. Pelletier. chef du contentieux de la Cité de Québec, prétend que le règlement sur cette matière adopté par la Cité de Québec le 23 janvier 1866, est encore en vigueur parce qu'il est l'exercice des pouvoirs concédés par la Charte de la Cité de Québec et non par la Loi des maîtres et serviteurs.
Comme conséquence, ces dispositions ont perdu leur caractère de droit public et sont devenues du droit supplétif; elles n'ont donc etfet qu'à défaut de stipulations contractuelles.

Le délai-congé se trouve aujourd'hui règlementé principalement ${ }^{1 *}$ par les dispositions expresses ou inplicites du contrat de travail (C.c. a. 1016).

Comme les dispositions du règlement d'atelier, ce que Me Beaulac appelle usage, forment partie du contrat d'engagement, la règlementation y prévue sur le délai-congé sera obligatoire pour les salariés qu'elle lie. Ainsi, dans certains établissements industriels, les salariés, aux termes du règlement interne, peuvent être congédiés à une heure d'avis.

L'intégration dans le domaine contractuel de la règlementation sur le délai-congé produit d'autres conséquences. Le délai-congé devient une condition de travail qui peut être règlementée par une ordonnance de la Commission du salaire minimum ${ }^{19}$ ou par un décret émis sous le régime de la Loi de la convention collective ${ }^{20}$; de telles règlementations ont, suivant ces statuts, un caractère de droit public.

Enfin, tout comme en France, le délai-congé peut maintenant faire l'objet des dispositions de la convention collective d'ordre privé, soit celle prévue à la Loi des syndicats professionnels, soit celle visée à la Loi des relations ouvrières ou au Code fédéral. Cette matière, comme d'autres, pourra dorénavant être l'objet de négociations entre un employeur et l'association accréditée de ses salariés, et être soumise à la considération du conseil d'arbitrage constitué sous le régime de la Loi des différends ouvriers de Québec ou encore sous celui de la loi sur les relations industrielles (fédérale).

(18) Si l'on admet que les règlements municipaux ont été abrogés par le statut $13 \mathrm{Geo}$. VI, c. 69.

(19) S.R.Q. 1941. c. 164 , a. 13

(20) S.R.Q., 1941, c. 163, a. 10. 